\title{
BOOSTER'S COUPLED BUNCH DAMPER UPGRADE
}

\author{
W. A. Pellico*, D. W. Wildman, \\ FNAL $^{\circ}$, Batavia, IL 60510, USA
}

\section{Abstract}

A new narrowband active damping system for longitudinal coupled bunch $(\mathrm{CB})$ modes in the Fermilab Booster has recently been installed and tested. In the past, the Booster active damper system consisted of four independent frontends. The summed output was distributed to the $18, \mathrm{~h}=84$ RF accelerating cavities via the RF fan-out system. There were several problems using the normal fan-out system to deliver the longitudinal feedback RF. The high power RF amplifiers normally operate from $37 \mathrm{MHz}$ to $53 \mathrm{MHz}$ whereas the dampers operate around $83 \mathrm{MHz}$. Daily variations in the tuning of the RF stations created tuning problems for the longitudinal damper system. The solution was to build a dedicated narrowband, $\mathrm{Q} \approx 10$, $83 \mathrm{MHz}$ cavity powered with a new $3.5 \mathrm{~kW}$ solid-state amplifier. The cavity was installed in June 2002 and testing of the amplifier and damper front-end began in August 2002.

A significant improvement has been made in both operational stability and high intensity beam damping. At present there are five CB modes being damped and a sixth mode module is being built. The new damper hardware is described and data showing the suppression of the coupled-bunch motion at high intensity is presented.

\section{HISTORY}

For almost three decades, longitudinal coupled-bunch motion in the Fermilab Booster has been a limiting factor in determining the extracted beam's longitudinal emittance. Various methods of damping the coupledbunch oscillations have been developed to keep pace with the steadily increasing proton intensities in the machine. The Booster coupled-bunch oscillations were first observed at a proton intensity of $1.2 \mathrm{E} 12 \mathrm{ppp}$. The two dominant modes near $\mathrm{m}=16$ and $\mathrm{m}=36$ (for $\mathrm{h}=84$, mode 34,35 and 36 is the same as mode 50,49 and 48) were correctly identified in 1977 [1] but were not associated with any particular RF cavity mode or other resonator in the machine. The solution at that time was to create a bunch by bunch spread in synchrotron periods by operating one of the $18 \mathrm{~h}=84 \mathrm{RF}$ cavities at a harmonic number of $\mathrm{h}=83$. This increased Landau damping, successfully reducing the coupled bunch motion.

By 1993 the $m=16$ and $m=36$ modes were identified with three specific resonances in the RF cavities [2,3]. The modes near $\mathrm{m}=16$ correspond to the $3^{\text {rd }}$ and $5^{\text {th }}$ "harmonics" of the Booster cavity at $165 \mathrm{MHz}$ and 217 $\mathrm{MHz}$. Both modes move in frequency as the bias on the

\footnotetext{
*Email: pellico@fnal.gov

- Operated by the URA, under contract No. DE-AC02$76 \mathrm{CH} 03000$ with the U.S. DOE.
}

cavity ferrite tuners changes during acceleration. Modes near $\mathrm{m}=36$ correspond to a non-tuning $(<30 \mathrm{kHz}$ shift $) \mathrm{RF}$ cavity mode near $83 \mathrm{MHz}$. The $\mathrm{m}=36$ mode frequency, primarily determined by the physical length of the RF cavity drift tube, has both acceleration gap voltages oscillating in phase. Once the offending modes were identified, two passive dampers were installed in each Booster RF cavity. These passive dampers eliminated the $\mathrm{m}=16$ line and decreased the $\mathrm{m}=36$ line by about an order of magnitude. This resulted in stable bunch motion and a factor of three reduction in the longitudinal emittance of the extracted beam at an intensity of 1.9E12 ppp. Further reduction in the $m=36$ mode line was accomplished by reducing the RF bucket area during acceleration to increase Landau damping [4] and by using a narrowband active damper at mode $\mathrm{m}=35$ to drive a wideband kicker [5].

The narrowband damper approach was later extended to include modes 1, 34,35, and 36 [6]. In this instance, the low level damping signal was applied to the beam through the $18, \mathrm{~h}=84 \mathrm{RF}$ cavities. Using the $18 \mathrm{RF}$ cavities as kickers had the disadvantage that the RF power amplifiers when driven around $83 \mathrm{MHz}$ were being operated well outside their normal operating frequency range of 30 to 53 MHz. A more serious drawback of this method was that the RF fanout is optimized to deliver properly phased RF signals at $\mathrm{h}=84$, not at $83 \mathrm{MHz}$. The amplitude and phase of the damping kick delivered to an individual proton bunch relied on the net vector sum of all 18 cavities at 83 $\mathrm{MHz}$. This system required frequent manual tuning of the low level damping signal and proved to be difficult to operate on a daily basis. For example, if one of the $18 \mathrm{RF}$ cavities failed or had a reduced output, the amplitude and phase of the damping kick would no longer be correct and both the gain and phase of the low level damping drive signal would have to be re-adjusted. The gain of the active system was adequate when Booster was below $4 \mathrm{E} 12$ protons per pulse, the present Booster intensities are now around 5E12 with higher intensities planned.

To alleviate these problems, a dedicated longitudinal kicker cavity was installed in the Booster to damp the coupled-bunch modes near $m=36$. The individual $R F$ cavities have a range of resonances between $82.0 \mathrm{MHz}$ and $82.8 \mathrm{MHz}$ with an average value of $82.45 \mathrm{MHz}$. The Q of these cavity modes was initially $\approx 3300$ but was reduced to $\approx 170$ by the addition of the passive dampers. To reduce the necessary drive power but still maintain a conservative design, the new $\mathrm{CB}$ damper cavity has a center frequency of $82.6 \mathrm{MHz}$ and a loaded $\mathrm{Q}=11$. 


\section{DAMPER FRONT END ELECTRONICS}

The previous $\mathrm{CB}$ damper system used a direct digital synthesizer (D.D.S) that could be clocked up to $800 \mathrm{MHz}$. This allowed a clock that was eight times the Booster VCO frequency (422 MHz.) to be used [4]. The D.D.S's provided quadrature outputs to be mixed with the beam signal band-passed filtered at $83 \mathrm{Mhz}$. Each mode module did it's own front end processing. This required each mode module to do it's own frequency transposition around $83 \mathrm{MHz}$. The new CB design has a single module (CB front-end module) that downconverts the beam signal to baseband then splits the output eight ways (we use only 5 at present.) With the common baseband error signal (approximately $21 \mathrm{MHz}$ ) the individual mode modules can use a D.D.S unit clocked at only twice the Booster VCO to do the necessary frequency transposition. The mode module outputs are then summed, upconverted, filtered and pre-amplified in the front-end module. The correction signal is then applied to a $3.5 \mathrm{KW}$ amplifier (see next section.) The layout of the new system is shown in Fig 1. The mode modules are similar to the old design with superheterodyne and single side-band mixing allowing for detection and phase correction of the $\mathrm{CB}$ mode error. The modules all have internal wide dynamic range phase shifters.

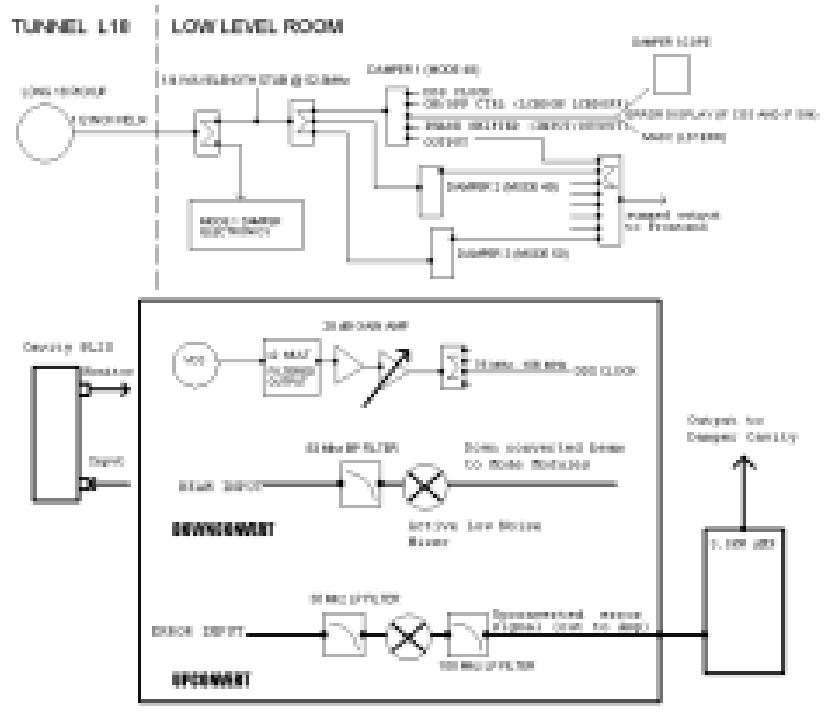

Figure 1. CB Damper Layout

\section{DAMPING CAVITY}

Figure 2 is a drawing of the new damping cavity. It is a single gap, quarter wave, coaxial aluminum resonator with an inner conductor diameter of $4.5^{\prime \prime}$ and an outer conductor diameter of $10^{\prime \prime}$. The center frequency is 82.6 $\mathrm{MHz}$. The loaded Q value of 11 is obtained by overcoupling the power amplifier drive loop to the cavity. A
4" diameter, 4" long ceramic gap was chosen so that the cavity would be operated below the calculated multipacting threshold of $4.3 \mathrm{kV}$. The ceramic gap is welded to a 4" diameter stainless steel beam pipe that slides through the center conductor of the cavity. The electrical connection between the aluminum cavity and the stainless beam pipe is made with two strips of $\mathrm{BeCu}$ finger stock.

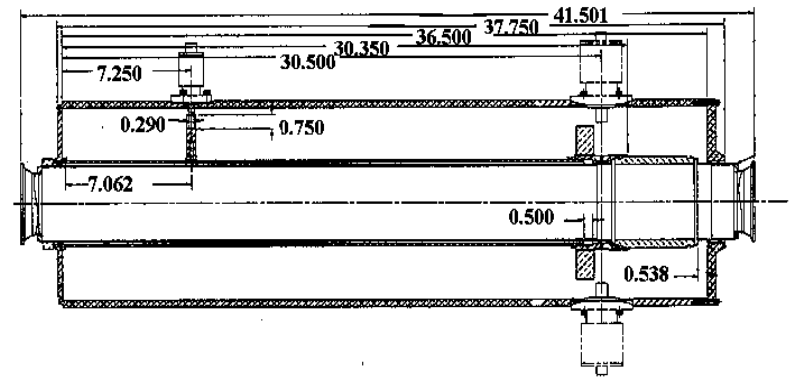

Figure 2. $83 \mathrm{MHz}$ Damping Cavity

To reduce the impedances of higher order modes (HOM's) in the damping cavity, a single 8" OD X 5" ID $X$ 1" thick ferrite core (Stackpole Ceramag 2285) is inserted around the cavity's inner conductor, $2.5^{\prime \prime}$ from the accelerating gap. The 2285 core has a Curie temperature of $510^{\circ} \mathrm{C}, \mu^{\prime}=12.5$ and a $\mathrm{Q} \approx 150$ at $83 \mathrm{MHz}$ which drops sharply above $120 \mathrm{MHz}$. The unloaded cavity Q is 268 and the shunt impedance is $15.9 \mathrm{k} \Omega$ with the input coupling loop disconnected from the drive-line. The HOM frequencies and loaded Q values are: $233 \mathrm{MHz}$ $(\mathrm{Q}=55.5), 251.3 \mathrm{MHz}(\mathrm{Q}=28)$, and $521.2 \mathrm{MHz}(\mathrm{Q}=70.8)$.

The damper cavity is driven by an Amplifier Research 3500A100 solid-state amplifier (maxium output power greater than 3500 watts at $83 \mathrm{MHz}$.) The RF power is transmitted to the cavity through $74.5 \mathrm{~m}$ of $7 / 8^{\prime \prime}$ Heliax coaxial cable with $\approx 1 \mathrm{~dB}$ attenuation. A maximum cavity peak gap voltage of 3670 volts was measured using a capacitively coupled (1000:1) gap monitor. This value is in good agreement with the measured forward and reflected power into the cavity from a dual directional coupler inserted in the $7 / 8^{\prime \prime}$ coaxial line.

\section{RESULTS}

The new damping cavity was installed in June 2002 and the existing CB mode 34,35 and 36 signals were switched over from the Booster fanout to the new amplifier and cavity. Mode 33 and 37 modules were built and tested several months later using the new front end module. The beam measurements shown in this paper are with the new electronics, cavity and $3.5 \mathrm{KW}$ amplifier damping $\mathrm{CB}$ modes 34,35 and 36 . A phase signal generated by mixing the signal from a wall current monitor in the MI- 8 line (MI-8 line is the extraction line from Booster to Main Injector/MinibooNE) with the Booster RF is used as a 
measure of the Booster's beam quality. Figure 3 is a display of the extracted beam's phase measurement with dampers off and on.

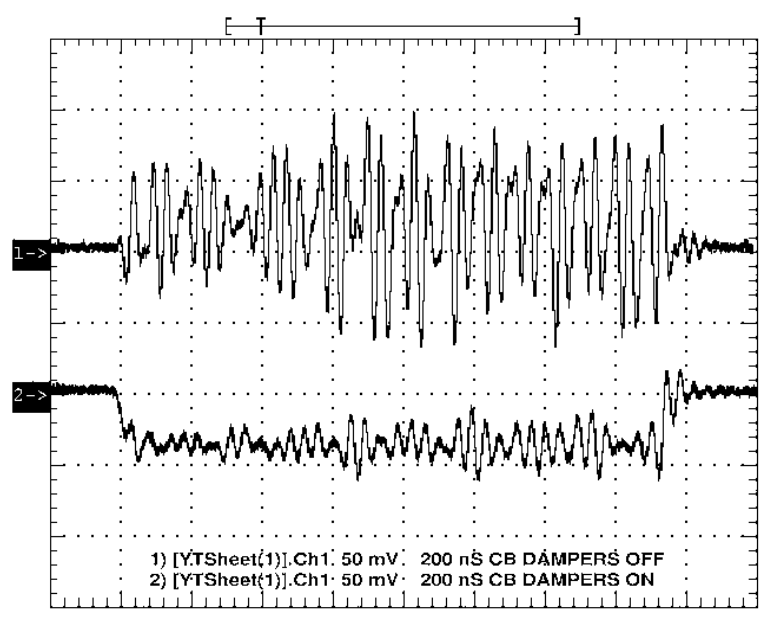

Figure 3. Scope Display of Phase 1)CB Off 2)CB

With dampers off, beam loss in the high dispersion regions of the MI-8 line will trip the Mini-BooNE ${ }^{\bullet}$ beam permit. Figure 4 shows the loss pattern on two downstream loss monitors with the dampers off/on. Booster CB dampers also play a critical role in the proton intensities used in colliding beams.

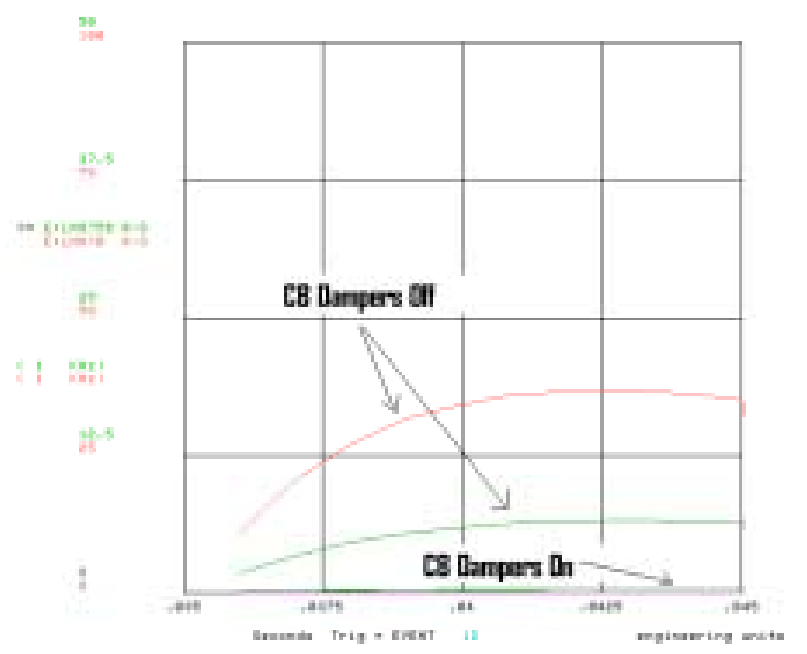

Figure 4. Loss Monitors in MI-8 Line: CB OFF/ON

The Main Injector proton coalescing efficiency/intensity is greatly improved (intensity dependent) when the dampers are on. The large dipole oscillations associated with bunch to bunch phase oscillations create an effective longitudinal emittance blowup. This problem also impacts performance in the Main Injector cycle used for making antiprotons.

\section{CONCLUSION}

Higher Booster proton intensities have required $\mathrm{CB}$ damper upgrades. The success of this $\mathrm{CB}$ damper system allows Booster to meet current proton demands. However, the requested proton intensity will be required to increase. The Booster $\mathrm{CB}$ system will require additional damping power and additional mode modules. The new modules are being built, as is a new damper cavity. The additional cavity will be used to damp mode 16. The plan is to install the new cavity sometime this summer.

\section{REFERENCES}

[1]C.M. Ankenbrandt, J.E.Griffin, R.P. Johnson, J. Lackey and K. Meisner, "Longitudinal Motion of the Beam in the Fermilab Booster," IEEE Trans. Nucl. Sci. NS-24 1449 (1977).

[2]D. Wildman and K. Harkay, "HOM RF Cavity Dampers for Suppressing Coupled Bunch Instabilities in the Fermilab Booster," 1993 PAC Proc. Washington, DC., p.3528A.

[3]K. Cecelia Harkay "A Study of longitudinal Instabilities and Emittance Growth in the Fermilab Booster Synchrotron." FERMILAB-THESIS-199365 (Dec 1993) 211p.

[4]J.P. Shaw, D. McGinnis, and R. Tomlin, "Reducing the Coupled-Bunch Oscillation in the Fermilab Booster by Optimizing RF Voltage," 1993 PAC Proc. Washington, DC. ,p.3787

[5]J.M. Steimel and D. McGinnis, "Damping in The Fermilab Booster," 1993 PAC Proc. Washington, DC., p. 2100

[6]D.A. Herrup, D. McGinnis, J. Steimel, and R. Tomlin, "Analog Dampers in the Fermilab Booster," 1995 PAC Proc. Dallas, Texas, p.3010

\footnotetext{
- The MiniBooNE experiment uses $8 \mathrm{GeV}$ protons from Booster to create a Neutrino beam.
} 